\title{
Molecular Simulation Study of Montmorillonite in Contact with Water
}

\author{
Yiteng Li, Arun Kumar Narayanan Nair*, \\ Ahmad Kadoura, Yafan Yang, and Shuyu Sun \\ Physical Science and Engineering Division (PSE), \\ Computational Transport Phenomena Laboratory, \\ King Abdullah University of Science and Technology (KAUST), \\ Thuwal, 23955-6900, Saudi Arabia.
}

November 21, 2018

*To whom correspondence should be addressed, email: arun.narayanannair@kaust.edu.sa 


\begin{abstract}
Grand canonical Monte Carlo and molecular dynamics simulations were applied to understand the molecular mechanism of ion and water transport in montmorillonite clays as a function of relative humidity $(\mathrm{RH})$. The variation of basal spacings of montmorillonite as a function of RH predicted based on the swelling free energy profiles was consistent with X-ray data. The hydration of the montmorillonite shows the following well-known order: $\mathrm{Mg}^{2+}>\mathrm{Ca}^{2+}>$ $\mathrm{Sr}^{2+}>\mathrm{Li}^{+}>\mathrm{Na}^{+}>\mathrm{K}^{+}$. The relative contribution of water on external surfaces only becomes significant close to the saturation pressure. However, this behavior for K-montmorillonite starts to occur well below the saturation pressure due to the clay-swelling inhibition by potassium ions. The diffusion of water and ions generally increases with $\mathrm{RH}$ in all samples. However, for samples with weakly hydrated ions, the water mobility in thin films adsorbed on external basal surfaces of clay can be higher than that in the water-saturated mesopores. For a given $\mathrm{RH}$, mobility of interlayer species is typically lower than that from the external surfaces. The results of the simulations were applied to interpret recent laboratory measurements of ion mobility with changing $\mathrm{RH}$. We also assess the effect of layer charge distribution on such sorption and diffusion processes.
\end{abstract}




\section{Introduction}

Smectite such as montmorillonite is one of the most common type of naturally occurring clay minerals that readily swell in the presence of water. ${ }^{1-25}$ Smectite clays are aluminosilicates comprised of negatively charged layers compensated by cations located in the interlayer region. Clay minerals play important roles in geologic sequestration of carbon dioxide, ${ }^{26-29}$ shale gas extraction, ${ }^{26,30-32}$ and nuclear waste disposal. ${ }^{33,34}$ For example, successful storage of $\mathrm{CO}_{2}$ is determined by the alterations in the permeability of overlying cap rock formations because of interaction with the contact fluid. Cap rocks generally consist of shale or mudstone enriched in clay minerals such as the smectite mineral montmorillonite. Notably, the extent to which shale formations rich in expandable clays swell is primarily controlled by $\mathrm{H}_{2} \mathrm{O}$ contents in the contact fluid. Understanding the fundamentals of clay-water interaction is crucial to the success of such applications. Moreover, the clay interlayer provides a distinct structural environment for investigating the role of confinement on sorption and diffusion processes.

A number of experimental ${ }^{1-13}$ and simulation ${ }^{14-25}$ studies have been carried out to determine the swelling properties of montmorillonite systems. These studies typically showed two regimes of the swelling process in montmorillonite due to water uptake: (a) Crystalline swelling at basal $d$-spacings below $\approx 19-21 \AA$ and (b) Osmotic swelling corresponding to basal $d$-spacings above $\approx 30 \AA$. The stable basal $d$-spacings are usually in the range $9.6-10.4 \AA$ for dry $(0 \mathrm{~W})$ montmorillonite. In the presence of water, the basal $d$-spacings increase and are in the range 11.5-12.5 $\mathrm{A}$ for a monolayer $(1 \mathrm{~W})$ water arrangement. The next stable state is a bilayer $(2 \mathrm{~W})$ with basal $d$-spacings in the 14.5-15.5 $\AA$ range, followed by three water layers $(3 \mathrm{~W})$ with basal $d$-spacings in the 18.0-19.1 A range. Additional water uptake, for example, induces transition from such crystalline swelling to the so-called osmotic swelling, where the basal $d$-spacing continuously increases with water content. The relative stabilities of these hydration states in the clay system can be determined from the minima in the swelling free energy profile. ${ }^{18,20}$ 
Several factors influence the sorption and transport properties of, e.g., water in smectite clay minerals such as relative humidity $(\mathrm{RH})$, pore space, interlayer cation, and layer charge distribution. ${ }^{1-21}$ Ferrage et al. ${ }^{8,9}$ studied hydration of montmorillonite as a function of $\mathrm{RH}$ by modeling of X-ray diffraction (XRD) patterns. They also investigated the effects of interlayer cation and layer charge distribution on the swelling mechanism of montmorillonite clays. Tambach et al. ${ }^{20}$ used simulations to study the molecular mechanism of clay swelling hysteresis as a function of RH. TeichMcGoldrick et al. ${ }^{21}$ applied molecular simulations to investigate the effects of interlayer cation, layer charge location, and temperature on the swelling properties of clay minerals. Such factors are important in determining the diffusion rates of various interlayer cations and water. ${ }^{10,13-19}$ Malikova et al. ${ }^{10}$ studied the diffusion mechanism of water in montmorillonite clays at low hydration by neutron spin-echo and time-of-flight techniques. The interpretation of experimental conductivity values provided the diffusion coefficients for interlayer cations as a function of $\mathrm{RH} .{ }^{13}$ Simulations of montmorillonite clays with varying $\mathrm{RH}$ reported so far have been restricted to adsorption studies. ${ }^{20,21}$ More recently, simulations were used to understand the molecular mechanism of the mobility of water and monovalent ions in mesopores of montmorillonite as a function of RH. ${ }^{35}$ However, a detailed understanding of the factors which influence the sorption and transport properties of water and ions in smectite clay minerals such as RH, pore space, interlayer cation, and layer charge distribution is still lacking.

Computer simulations are very suitable to study the organization and dynamics of complex systems. ${ }^{36-41}$ In this study, grand canonical Monte Carlo (GCMC) simulations were carried out to obtain molecular-level understanding of the influence of RH on water uptake by montmorillonite at $298.15 \mathrm{~K}$. The focus of this study is on Wyomingtype montmorillonite comprising of tetrahedral silica and octahedral alumina sheets that coordinate to form a 2:1 or tetrahedral-octahedral-tetrahedral layer. Furthermore, these simulation samples are saturated with monovalent $\left(\mathrm{Li}^{+}, \mathrm{Na}^{+}\right.$, or $\left.\mathrm{K}^{+}\right)$and divalent $\left(\mathrm{Mg}^{2+}, \mathrm{Ca}^{2+}\right.$, or $\left.\mathrm{Sr}^{2+}\right)$ ions. The stable basal $d$-spacing values determined from the free energy profiles and the water uptake as computed from simulations were 
used to aid in the interpretation of clay swelling experiments. ${ }^{3-8,11}$ Then, molecular dynamics (MD) simulations were applied to understand the molecular mechanism of ion and water diffusion in montmorillonite as a function of RH. An overall goal of our research is to apply these simulations to interpret the experimental measurements ${ }^{10,13}$ of self-diffusion coefficients of ions and water in the clay interlayers as a function RH. Also, sorption and diffusion properties of interlayer species as a function of $\mathrm{RH}$ are compared with those from external surfaces. Finally, we report the effect of layer charge distribution on such sorption and diffusion processes.

\section{Simulation details}

All GCMC and MD simulations are carried out with TOWHEE ${ }^{42}$ and LAMMPS $^{43}$ packages, respectively. Since the molecular model and the simulation method used here were similar to those presented in our earlier works, ${ }^{38-41}$ we briefly outline the simulation procedure. Our simulations use clay model based on the pyrophyllite unit cell structure $\left(\mathrm{Si}_{8} \mathrm{Al}_{4} \mathrm{O}_{20}(\mathrm{OH})_{4}\right)$. The supercell of orthorhombic symmetry has 64 unit cells (2560 atoms) constituting the mineral portion of the clay phase (Fig. 1). That is, each of the two parallel clay layers has dimensions of $42.24 \times 36.56 \times$ $6.56 \AA$. The $z$ dimension of the simulation box $L_{z}=2 d$, where $d$ is the basal spacing which is the sum of the clay layer thickness $(6.56 \AA)$ ) and the interlayer region. We have focused our study on Wyoming-type montmorillonite of unit cell formula $\mathrm{M}_{0.75 / n}\left(\mathrm{Si}_{8}\right)\left(\mathrm{Al}_{3.25} \mathrm{Mg}_{0.75}\right) \mathrm{O}_{20}(\mathrm{OH})_{4}$, where $\mathrm{M}$ represents a counterion $\left(\mathrm{Li}^{+}, \mathrm{Na}^{+}, \mathrm{K}^{+}\right.$, $\mathrm{Mg}^{2+}, \mathrm{Ca}^{2+}$, or $\mathrm{Sr}^{2+}$ ) and $n$ is the counterion valency. According to this formula, each clay sheet accommodates 24 isomorphic substitutions of $\mathrm{Al}$ by $\mathrm{Mg}$ ion in the octahedral sheet and 24 (12) compensating monovalent (divalent) counterions in the interlayer region. All these substitutions follow the restrictions of Loewenstein's rule (e.g., replacement sites cannot be adjacent to each other). The clay model was assumed to be rigid during our simulations. Furthermore, we employed periodic boundary conditions in three directions. 
The interactions between particles were assumed to be pairwise additive and are modeled based on the Lennard-Jones (LJ) 12-6 potential. ${ }^{44}$ The Lorentz-Berthelot mixing rule was used to determine interactions between particles with different LJ parameters. The charged atoms interact with one another via the direct Coulomb potential. The electrostatic interactions were treated using the Ewald summation (GCMC) or particle-particle particle-mesh (MD) method with a precision value of $10^{-5}$. Force field parameters for clay are as in CLAYFF. ${ }^{45}$ Water was modeled by the simple point charge (SPC) water having flexible intramolecular interactions ${ }^{45}$ (GCMC simulations used rigid $\mathrm{SPC}^{46}$ model for water). The LJ parameters of the mobile $\mathrm{Li}^{+},{ }^{47}$ $\mathrm{Na}^{+},{ }^{18} \mathrm{~K}^{+},{ }^{48} \mathrm{Mg}^{2+},{ }^{47} \mathrm{Ca}^{2+},{ }^{48}$ and $\mathrm{Sr}^{2+47}$ ions are are taken from the literature. The LJ parameters and charges used in this study are presented in Table S1, Supporting Information.

GCMC simulation was used to study the adsorption of water by montmorillonite in the $\mu_{\mathrm{H}_{2} \mathrm{O}} V T$ ensemble, where $\mu_{\mathrm{H}_{2} \mathrm{O}}$ is the chemical potential of water. The imposed $\mathrm{H}_{2} \mathrm{O}$ chemical potential corresponds to a pressure of $\mathrm{RH} \times P_{0}$, where $P_{0}$ represents the saturated vapor pressure of the SPC model (about 0.032 bar $^{49,50}$ at $298.15 \mathrm{~K}$ in good agreement with the experiments). Each GCMC simulation typically consisted of about $4 \times 10^{7}$ Monte Carlo steps for the equilibration phase and $2 \times 10^{7}$ steps for the production phase. The swelling states of the clay model are obtained using the stability analysis based on the normal pressure (output from TOWHEE) and the swelling free energy. ${ }^{18,20}$ The final conformations outputted by our GCMC simulations were employed as the initial conformations in the MD simulations. Equilibration runs of 1 ns were carried out in the $N V T$ ensemble, followed by 4 ns production runs in the $N V E$ ensemble. Three independent trajectories each of length 5 ns per simulation are computed to achieve good statistical averages. The equations of motion are integrated using the velocity Verlet algorithm with a time step of 1 fs. The temperature is controlled by a Nośe-Hoover thermostat ${ }^{44}$ using a relaxation time of 0.1 ps and a drag value of 1.0. The nonbond terms were handled with a cutoff at $9.5 \AA$. The long-range van der Waals interactions are included via tail corrections. The differences of system temperatures 
from the preset value during $N V E$ production runs are mostly negligible (typically $<1 \%)$.

\section{Results and discussion}

\subsection{Swelling curves}

GCMC simulations were carried out to understand the swelling process of montmorillonite as a function of RH. Oscillations in the pressure provide a signature for crystalline swelling processes and a means for distinguishing integer-layer hydrate states. ${ }^{15,20,51}$ The simulation results for pressure and swelling free energy profiles of all samples are shown in Figs. S1 and S2, Supporting Information. Each free energy curve displays distinct minima that correspond to points where the normal pressure crosses the applied pressure line with negative slope. ${ }^{15}$ The applied pressure originates from both the bulk pressure, as determined by the temperature and chemical potential of the reservoir, and from any external pressure. The partial water-vapor pressure is the applied pressure in our simulations. Furthermore, Fig. 2 shows the basal spacing values at the global energy minima as computed from simulations (symbols) and the corresponding experimental data (lines). The experimental results are taken from Ferrage et al. ${ }^{8}$ Other available experimental results ${ }^{3-7,11}$ are in agreement with the ones plotted here and not included for clarity. We find good agreement between our simulation results and the experimental data. The experimental swelling curves may not identify the equilibrium states since an adsorption/desorption hysteresis loop occurs for swelling of the clay. ${ }^{8,23}$ The simulation results enclosed by the experimental hysteresis data may establish the true equilibrium clay layer spacings. ${ }^{23}$ The simulated layer spacings reported here are in good agreement with those determined by Boek et al. ${ }^{23}$ Therefore, the global minima in our simulated swelling free energy curves may provide the true equilibrium clay layer spacings of the system. With the aim of gaining more insight into the swelling process, we plot in Fig. 3 water uptake by montmorillonite as 
computed from simulations (open symbols) and the corresponding experimental data (lines). These experimental results are taken from Ferrage et al. ${ }^{8}$ Other available experimental results ${ }^{3-7,11}$ are in agreement with the ones plotted here and not included for clarity. Note that for simplicity and in accordance with other studies, ${ }^{21,40}$ we used basal distances $d=10.0,12.0$, and $15.0 \AA$ in our simulations representing, e.g., the swelling states $\approx 0 \mathrm{~W}, \approx 1 \mathrm{~W}$, and $\approx 2 \mathrm{~W}$, respectively. Table 1 provides approximate $\mathrm{RH}$ ranges and the corresponding basal distances/hydration states, for example, observed experimentally ${ }^{8}$ and used in our simulations of montmorillonite in contact with water (see also Fig. 2). The simulation results of water uptake are in good qualitative agreement with the experimental data. The discrepancy here may arise from minor differences in layer charge and/or basal $d$-spacing between simulation and experiment as well as force field issues. Other possible reasons for this discrepancy may include framework flexibility, registry motion of clay sheets, ${ }^{15}$ and turbostratic stacking of clay layers $^{52}$ which are not considered in this study. Our results are also consistent with previous simulation studies $^{21}$ (solid symbols in Fig. 3). Additionally, density profiles of different interlayer species estimated along the $z$-axis (perpendicular to the montmorillonite surface) in relevant hydration states close to the free energy minima are shown in Figs. S3 and S4. Note that as a result of the difference between the simulation and experimental results of water adsorption (see Fig. 3), the present results are at least qualitatively applicable.

Our results show that the swelling tendency of montmorillonite is related to the hydration energy of the ions. The hydration of montmorillonite is following the wellknown order: $\mathrm{Mg}^{2+}>\mathrm{Ca}^{2+}>\mathrm{Sr}^{2+}>\mathrm{Li}^{+}>\mathrm{Na}^{+}>\mathrm{K}^{+}$. We observe crystalline swelling where the stable basal $d$-spacings are in the range of about 9.6-10.4 $\AA$ for dry $(0 \mathrm{~W})$ montmorillonites and grow in the presence of water to the range of about 11.5-12.5 ^ making a monolayer (1W) water structure. The next stable state is a bilayer $(2 \mathrm{~W})$ in the range of about 14.5-15.5 $\AA$. Under conditions close to or above water saturation, water can further form such as three layers $(3 \mathrm{~W})$ with basal $d$-spacings in the range of about $18.0-19.2 \AA^{12}$ or induce transition from crystalline swelling to the 
so-called osmotic swelling, where the basal d-spacing increases continuously with water content. ${ }^{1,2}$ Our simulations show that a typical sample at $100 \%$ RH contains about $14 \mathrm{H}_{2} \mathrm{O}$ molecules per $\mathrm{O}_{20}(\mathrm{OH})_{4}$ in the $3 \mathrm{~W}$ state (basal $d$-spacing of $18 \AA$ ) which is in agreement with previous measurements. ${ }^{12,19}$ The $3 \mathrm{~W}$ state or the swelling of clays for $\mathrm{RH} \gtrsim 100 \%$ is not further considered in this study. Similar swelling behavior of clay was reported by ab initio molecular dynamics (AIMD) simulations in variably hydrated Ca-montmorillonite. ${ }^{22}$ AIMD studies showed that initially the interlayer space expands approximately $20 \%$ compared to the dry clay and higher water content systems indicate about $27 \%$ expansion compared to the $1 \mathrm{~W}$ state. Interestingly, we see that the change in $\mathrm{RH}$ hardly affects the sorbed amount of $\mathrm{H}_{2} \mathrm{O}$ and the distribution of different species in each hydration state. Note that the smooth increase in the adsorbed water amount observed in the experiments ${ }^{3-7,11}$ appears to be in disagreement with the corresponding stepwise change discussed above. This difference may be described based on the hydration-heterogeneity model that suggests the coexistence of integral hydrations states at a fixed RH. ${ }^{8}$ However, the investigation of such complex processes is out of scope of this work. Also, pronounced variations are obtained for the density profiles of counterions with increasing $\mathrm{RH}$, mostly because of the expansion of the clay. The adsorption of counterions onto clay leads to both inner- and outer-sphere surface complexes, which is in agreement with earlier simulations. ${ }^{18,24}$ An inner-sphere surface complex has no water molecule present between the surface functional group and the cation it binds, whereas an outer-sphere surface complex has at least one water molecule interposed between the cation and the surface. ${ }^{53}$ The monovalent counterions show significant population in the inner-sphere complexes and the divalent counterions exist mostly in the outer-sphere configurations. This trend is consistent with the observation that increasing hydration energy of the cations preclude the formation of inner-sphere configurations. ${ }^{18,24}$

Furthermore, the simulated in-plane $(x y)$ density distributions of molecules in the clay interlayers are given in Figs. S5-S15. The overall distribution behavior was very similar to that obtained in the previous simulation studies. ${ }^{14,15,17,40}$ For example, coun- 
terions present in the inner-sphere configurations are typically correlated with substitutions in the octahedral sheet. Our results show that water molecules are essentially associated with the interlayer ions and water can extend over the whole surface forming patterns at high RH. The orientation of interlayer water with respect to the normal vector of the closest clay platelet is shown in Figs. S16-S21. The overall orientation behavior was also very similar to that observed in the previous simulation works. ${ }^{20,40}$ We see that the favored angles for the water dipoles near a given surface are $\approx 90^{\circ}$ and $\approx 135^{\circ}$ in the one-layer and two-layer water systems, respectively. Notably, the $\mathrm{H}-\mathrm{H}$ vector does not lie parallel to the clay surface in the one-layer water system with monovalent counterions.

\subsection{Water films on external surfaces of montmorillonite}

GCMC simulations were also carried out to understand water adsorption onto external surfaces (basal $d$-spacing fixed at $65 \AA$ ) of montmorillonite as a function of RH. Fig. 4 shows the amount of water adsorbed onto external surfaces of montmorillonite as computed from simulations (symbols). Our results are compared with the Brunauer, Emmett, and Teller (BET) ${ }^{54}$ adsorption isotherm model (lines) which provide complementary multilayer adsorption analysis of water uptake on external clay surfaces. The simulation results are in good qualitative agreement with the BET theory. Table S2 reports the BET fitting parameters for all clay samples. As expected, samples saturated with divalent cations display more significant water adsorption over the entire range of $\mathrm{RH}$ values studied when compared to those samples containing monovalent cations. For example, based on the BET analysis, the monolayer coverages of montmorillonite with monovalent and divalent cations are about 4 and $7 \mathrm{H}_{2} \mathrm{O}$ molecules per $\mathrm{O}_{20}(\mathrm{OH})_{4}$, respectively. As noted previously, ${ }^{11} \mathrm{BET}$ analysis fails to adequately describe adsorption phenomena at $\mathrm{RH}$ values close to saturation. Comparing the amount of water determined by modeling the X-ray diffraction patterns with that obtained from the gravimetry experiments may allow discriminating the relative contributions of $\mathrm{H}_{2} \mathrm{O}$

molecules from interlayers and pore space network. ${ }^{8}$ An important finding is that at 
identical conditions the relative contribution of $\mathrm{H}_{2} \mathrm{O}$ on external surfaces only becomes significant close to the saturation pressure (compare, e.g., Figs. 3 and 4). However, this behavior for K-montmorillonite starts to occur well below the saturation pressure due to the clay-swelling inhibition by potassium ions.

Furthermore, density profiles of water and ions located at the external surfaces of montmorillonite as a function of RH are shown in Figs. S22-S23. Under fully saturated conditions, water shows bulk-like behavior away from the clay surface $(z \gtrsim 10 \AA)$ in all cases. Three strongly structured water layers at $z \approx 3,6$, and $9 \AA$ can be identified in these density profiles. The positions of maxima and minima and their magnitude obtained here are in excellent agreement with previous simulation studies of water behavior in clay mesopores. ${ }^{35,55}$ Here also the ions have a strong preference to form outer-sphere surface complexes with increasing $\mathrm{RH}$ and/or ion hydration energy, consistent with other reports. ${ }^{35}$ The density minima separating the first and the second water layers coincide with the maxima in the ion density profiles corresponding to the outer-sphere surface complexes. As the water content is increased to saturation, ions are seen to shift gradually from outer-sphere surface complexes to diffuse-layer species.

\subsection{Diffusion of the interlayer species in montmorillonite}

The self-diffusion coefficients have been estimated from the linear slope of the mean square displacements of the species versus time, and the results are provided in Table S3. Overall, the calculated diffusion coefficients of ions and water are in reasonable agreement with experiments (see Table S3). ${ }^{10,13}$ Note that a relatively high amount of water, e.g., in the simulated $2 \mathrm{~W}$ state (see Fig. 3) might result in slightly lower mobility of interlayer species than experimental values. The influence of framework flexibility on diffusion coefficients increases as water content decreases which is evident from comparison of our results with those of Greathouse et al. ${ }^{19}$ Similar behavior has been reported by Holmboe and Bourg. ${ }^{56}$ All these self-diffusion coefficients were normalized by the corresponding bulk values at about $298.15 \mathrm{~K}$ and given in Fig. 5. The bulk diffusion coefficients of $\mathrm{Li}^{+}, \mathrm{Na}^{+}, \mathrm{K}^{+}, \mathrm{Mg}^{2+}, \mathrm{Ca}^{2+}, \mathrm{Sr}^{2+}$, and $\mathrm{H}_{2} \mathrm{O}$ are about 1.03, 1.33, 
$1.96,0.71,0.79,0.79$, and $2.30 \times 10^{-9} \mathrm{~m}^{2} / \mathrm{s}$, respectively. ${ }^{57,58}$ Here each bulk phase was approximated by that of water-saturated mesopore. The resulting estimates (see below) of diffusion coefficients are close to these experimental values. ${ }^{57,58}$ As with the sorbed amount of $\mathrm{H}_{2} \mathrm{O}$ and the distribution of different interlayer species, the change in RH hardly affects the diffusion coefficients of different species in each hydration state. For all systems, the self-diffusion coefficient of water in the $1 \mathrm{~W}$ hydration state decreases by about an order of magnitude compared to its bulk counterpart. Mobility of an ion in the $1 \mathrm{~W}$ state is lower than that in the bulk by about an order of magnitude for monovalent case and two orders of magnitude for divalent case. The diffusion of all species typically increases with RH because of the associated swelling of the clay. Therefore, at high RH, sorbate diffusion is comparable to that in the bulk for all samples except K-montmorillonite in which potassium acts as a swelling inhibitor. For example, the simulated diffusion coefficient of $\mathrm{K}^{+}$is about $2.5 \times 10^{-11} \mathrm{~m}^{2} / \mathrm{s}$ independent of $\mathrm{RH}$, and the corresponding value obtained using other force fields was in the range of about $0.6-18.0 \times 10^{-11} \mathrm{~m}^{2} / \mathrm{s}^{25}$ Consistent with the results of solvation energies, ${ }^{59}$ the diffusion coefficients of water are generally higher in the hydration states of samples saturated with monovalent ions than with divalent ions.

\subsection{Surface diffusion in water film}

Self-diffusion coefficients of water and ions on the external surface of montmorillonite as a function of RH are given in Tables S4 and S5. All these self-diffusion coefficients were normalized by the corresponding bulk values and shown in Fig. 6. The current results agree well with previous simulation results for Na-montmorillonite. ${ }^{35}$ The ionic mobility monotonically increases with RH in all samples, which is the expected behavior for the shift in distribution from, e.g., inner- to outer-sphere complexes (see also Figs. S22S23). Consistent with this mechanism, as RH increases the number of surface oxygen sites in the first coordination shell of $\mathrm{Na}^{+}$decreases and water molecules become part of it. ${ }^{35}$ This is not always the case for water. For example, if the RH is not too low, the water mobility in thin water films is higher than that in the water-saturated system. 
We observe this enhancement in the water mobility for samples with weakly hydrated ions. The increased water mobility in thin films in comparison to saturated samples is due to the faster hydrogen bond dynamics at the liquid-vapor interface. ${ }^{60}$ At lower $\mathrm{RH}$, water molecules are associated with the surface-bound cations and thus become less mobile. Our results show that for a given RH, mobility of interlayer species is typically lower than that from the external surfaces (compare, e.g., Figs. 5 and 6). This may be attributed to the steric constraints of the interlayer regions. For example, the diffusion of each species in the $1 \mathrm{~W}$ state $(\approx 20 \% \mathrm{RH})$ is about an order of magnitude lower than that in the films. At high RH this difference in diffusion coefficients, as expected, becomes smaller for all samples except K-montmorillonite.

Overall, the diffusion properties of water and ions in the interlayers are controlled by the swelling state with the global energy minimum. Our results show that ion hydration energy plays a critical role in determining the interlayer expansion of smectite clay minerals when exposed to water. Thus, among the investigated monovalent ions, mainly potassium ions showed clay swelling inhibition. This means that an interlayer species is relatively less mobile in K-montmorillonite at all $\mathrm{RH}$. While, among the investigated divalent ions, $\mathrm{Sr}^{2+}$ showed less swelling of the clay. Therefore, for divalent case, an interlayer species is relatively less mobile in Sr-montmorillonite at all RH. However, ion hydration energy has a much less drastic effect on the diffusion properties of water and ions on the external surfaces. For example, among the investigated monovalent ions, the water mobility associated with potassium ions at intermediate $\mathrm{RH}$ is relatively higher than that in the water-saturated mesopores.

\subsection{Influence of layer charge distribution}

Montmorillonite may exhibit limited tetrahedral substitutions. ${ }^{8,13,28,29}$ In order to understand the effect of layer charge distribution on sorption and diffusion processes, we also simulated the corresponding $\mathrm{M}_{0.75 / n}\left(\mathrm{Si}_{7.75} \mathrm{Al}_{0.25}\right)\left(\mathrm{Al}_{3.5} \mathrm{Mg}_{0.5}\right) \mathrm{O}_{20}(\mathrm{OH})_{4}$ case in the presence of water. This montmorillonite has both octahedral and tetrahedral substitutions. For simplicity, arrangement of isomorphic substitutions is based on the one used 
by Chávez-Páez et al. ${ }^{16}$ Here we compare the results obtained using this model with those obtained using $\mathrm{M}_{0.75 / n}\left(\mathrm{Si}_{8}\right)\left(\mathrm{Al}_{3.25} \mathrm{Mg}_{0.75}\right) \mathrm{O}_{20}(\mathrm{OH})_{4}$ (see above). We checked that the swelling process with respect to spacing and water content was not much affected by the layer charge distribution in all cases. The density profiles of different interlayer species computed along the $z$-axis in relevant hydration states close to the free energy minima are shown in Figs. S24 and S25. Note that, tetrahedral substitutions favor the formation of inner-sphere surface complexes here over outer-sphere species. The in-plane $(x y)$ density distributions of various interlayer species are shown in Figs. S26S36. We generally observe two types of inner-sphere complexes in one of which ions are associated exclusively with the negatively charged tetrahedral substitution sites. Furthermore, the orientation of interlayer water with respect to the normal vector of the closest clay platelet is hardly affected by charge distribution (Figs. S37-S42).

The amount of water adsorbed onto external surfaces of montmorillonite as computed from simulations (symbols) is compared with the BET model (lines) in Fig. S43. Table S6 reports the BET fitting parameters for all clay samples. In each case we find a decrease in adsorption capacity in the presence of tetrahedral substitutions. This decrease may be attributed to the fact that isomorphic substitution in the tetrahedral sheet of montmorillonite favors the formation of inner-sphere surface complexes over outer-sphere species (Figs. S44 and S45). The ion-water interaction is therefore becoming relatively more favorable in mesopores of montmorillonite with layer charge exclusively in the octahedral sheet.

Self-diffusion coefficients of interlayer water and ions as a function of RH are given in Table S7. Overall, the diffusion behavior of water is not much affected by the layer charge distribution. Mobility of ions in the $1 \mathrm{~W}$ state and, to a lesser extent, in the 2W state shows a marked decrease with substitutions in the tetrahedral layer. For example, the self-diffusion coefficients can vary by as much as an order of magnitude for divalent ions. This may be attributed to the presence of strong inner-sphere surface complexes associated with tetrahedral substitution sites. Self-diffusion coefficients of water and ions on the external surface of montmorillonite as a function of RH are given 
in Tables S8 and S9. Here also the diffusion coefficients of water on external surfaces are not much affected by the layer charge distribution, while those of ions are retarded.

\section{Conclusions}

Molecular simulations have been used to understand the molecular mechanism of water and ion transport in partially saturated clays. The basal spacing values at the free energy minima and the water uptake as computed from simulations are in good agreement with experimental measurements. ${ }^{8}$ The hydration of the montmorillonite follows the order, $\mathrm{Mg}^{2+}>\mathrm{Ca}^{2+}>\mathrm{Sr}^{2+}>\mathrm{Li}^{+}>\mathrm{Na}^{+}>\mathrm{K}^{+}$, suggesting that the hydration mechanism is dependent upon the interlayer ion hydration energy. Comparing the water uptake determined by modeling the X-ray diffraction results with that provided by the gravimetry experiments may allow discriminating the relative contributions of $\mathrm{H}_{2} \mathrm{O}$ molecules from interlayers and pore space network. ${ }^{8}$ While water molecules in the interlayer spaces and mesopores contribute to the total uptake, $\mathrm{H}_{2} \mathrm{O}$ sorption is likely dominated by uptake in the mesopores at high RH. Our simulations imply that, the relative contribution of $\mathrm{H}_{2} \mathrm{O}$ on external surfaces only becomes important close to the saturation pressure. This behavior for K-montmorillonite starts to appear, however, well below the saturation pressure due to the clay-swelling inhibition by potassium ions.

The diffusion of water and ions typically increases with RH in all investigated samples. However, for samples with weakly hydrated ions, the water mobility in thin films adsorbed on external clay surfaces can be higher than that in the water-saturated system. Recently Salles et al. ${ }^{13}$ reported diffusion coefficients of interlayer ions as a function of RH by combining electrical conductivity measurements and water adsorption. Our simulation results are in good agreement with these experimental values. These results indicate a significant variation in ionic mobility with $\mathrm{RH}$ and confirm the

impact of cation hydration on the overall process. In contrast, the diffusion behavior of weakly hydrated ions (e.g., $\mathrm{K}^{+}$) in the interlayers appears to be independent of RH. For 
a given RH, mobility of interlayer species is typically lower than that from the external surfaces which may be attributed to the steric constraints of the interlayer regions. Our studies also indicate that the limited tetrahedral substitutions in the montmorillonite clay mainly affect the ionic mobilities. Further simulations should be performed to investigate the influence of framework flexibility, layer charge, and geological conditions on clay swelling. Such studies have been reported for water-saturated clays. ${ }^{56,61}$ This approach will be extended to study samples at varying relative humidities in a future publication.

\section{Acknowledgments}

The research reported in this publication was supported by funding from King Abdullah University of Science and Technology (KAUST). Y. L., A. K. N. N., and Y. Y. gratefully acknowledge computational facilities provided at KAUST.

\section{Supporting Information}

Additional details of simulation analysis are provided in the Supporting Information. 


\section{References}

(1) Norrish K. The swelling of montmorillonite. Discuss. Faraday Soc. 1954, 18, $120-134$.

(2) Foster, W. R.; Savins, J. G.; Waite, J. M. Lattice expansion and rheological behavior relationships in water-montmorillonite systems. Clays Clay Miner. 1955, 3, 296-316.

(3) Keren, R.; Shainberg, I. Water Vapor Isotherms and Heat of Immersion of Na/CaMontmorillonite Systems-I: Homoionic Clay. Clays Clay Miner. 1975, 23, $193-$ 200.

(4) Fu, M. H.; Zhang, Z. Z.; Low, P. F. Changes in the properties of a montmorillonite-water system during the adsorption and desorption of water: Hysteresis. Clays Clay Miner. 1990, 38, 485492.

(5) Berend, I.; Cases, J.; Francois, M.; Uriot, J. P.; Michot, L. J.; Masion, A.; Thomas, F. Mechanism of Adsorption and Desorption of Water Vapor by Homoionic Montmorillonites: 2. The $\mathrm{Li}^{+}, \mathrm{Na}^{+}, \mathrm{K}^{+}, \mathrm{Rb}^{+}$, and $\mathrm{Cs}^{+}$-Exchanged Forms. Clays Clay Miner. 1995, 43 (3), 324336.

(6) Cases, J. M.; Berend, I.; Francois, M.; Uriot, J. P.; Michot, L. J.; Thomas, F. Mechanism of Adsorption and Desorption of Water Vapor by Homoionic Montmorillonite: 3. The $\mathrm{Mg}^{2+}, \mathrm{Ca}^{2+}, \mathrm{Sr}^{2+}$ and $\mathrm{Ba}^{2+}$ Exchanged Forms. Clays Clay Miner. 1997, $45(1), 822$.

(7) Dios Cancela, G.; Huertas, F. J.; Romero Taboada, E.; Sanchez-Rasero, F.; Hernandez Laguna, A. Adsorption of Water Vapor by Homoionic Montmorillonites. Heats of Adsorption and Desorption. J. Colloid Interface Sci. 1997, 185, 343354.

(8) Ferrage, E.; Lanson, B.; Sakharov, B. A.; Drits, V. A. Investigation of Smectite Hydration Properties by Modeling Experimental X-ray Diffraction Patterns: Part I. Montmorillonite Hydration Properties. Am. Mineral. 2005, 90, 1358-1374. 
(9) Ferrage, E.; Lanson, B.; Sakharov, B. A.; Geoffroy, N.; Jacquot, E.; Drits, V. A. Investigation of Dioctahedral Smectite Hydration Properties by Modeling of X-ray Diffraction Profiles: Influence of Layer Charge and Charge Location. Am. Mineral. 2007, 92, 1731-1743.

(10) Malikova, N.; Cadene, A.; Marry, V.; Dubois, E.; Turq, P. Diffusion of Water in Clays on the Microscopic Scale: Modeling and Experiment. J. Phys. Chem. B 2006, $110(7), 3206-3214$.

(11) Hatch, C. D.; Wiese, J. S.; Crane, C. C.; Harris, K. J.; Kloss, H. G.; Baltrusaitis, J. Water Adsorption on Clay Minerals as a Function of Relative Humidity: Application of BET and Freundlich Adsorption Models. Langmuir 2012, 28, 1790-1803.

(12) Dazas, B.; Ferrage, E.; Delville, A.; Lanson, B. Interlayer structure model of trihydrated low-charge smectite by X-ray diffraction and Monte Carlo modeling in the grand canonical ensemble. Am. Mineral. 2014, 99 (36), 17241735.

(13) Salles, F.; Douillard, J.M.; Bildstein, O.; El Ghazi, S.; Prelot, B.; Zajac, J.; Van Damme, H. Diffusion of Interlayer Cations in Swelling Clays as a Function of Water Content: Case of Montmorillonites Saturated with Alkali Cations. J. Phys. Chem. C 2015, 119 (19), 10370-10378.

(14) Chang, F.-R. C.; Skipper, N. T.; Sposito, G. Computer Simulation of Interlayer Molecular Structure in Sodium Montmorillonite Hydrates. Langmuir 1995, 11, 27342741.

(15) Young, D. A.; Smith, D. E. Simulations of clay mineral swelling and hydration: Dependence upon interlayer ion size and charge. J. Phys. Chem. B 2000, 104, 9163-9170.

(16) Chávez-Páez, M.; Van Workum, K.; De Pablo, L.; de Pablo, J. J. Monte Carlo simulations of Wyoming sodium montmorillonite hydrates. J. Chem. Phys. 2001, 114 (3), 1405-1413. 
(17) Marry, V.; Turq, P. Microscopic simulations of interlayer structure and dynamics in bihydrated heteroionic montmorillonites. J. Phys. Chem. B 2003, 107 (8), $1832-1839$.

(18) Whitley, H. D.; Smith, D. E. Free energy, energy, and entropy of swelling in Cs-, Na-, and Sr-montmorillonite clays. J. Chem. Phys. 2004, 120 (11), 5387-5395.

(19) Greathouse, J.A.; Cygan, R.T.; Fredrich, J.T.; Jerauld, G.R. Molecular Dynamics Simulation of Diffusion and Electrical Conductivity in Montmorillonite Interlayers. J. Phys. Chem. C 2016, 120 (3), 1640-1649.

(20) Tambach, T. J.; Bolhuis, P. G.; Hensen, E. J.; Smit, B. Hysteresis in clay swelling induced by hydrogen bonding: accurate prediction of swelling states. Langmuir 2006, 22 (3), 1223-1234.

(21) Teich-McGoldrick, S. L.; Greathouse, J. A.; Jove-Colon, C. F.; Cygan, R. T. Swelling properties of montmorillonite and beidellite clay minerals from molecular simulation: comparison of temperature, interlayer cation, and charge location effects. J. Phys. Chem. C 2015, 119 (36), 20880-20891.

(22) Lee, M. S.; McGrail, B. P.; Glezakou, V. A. Microstructural Response of Variably Hydrated Ca-rich Montmorillonite to Supercritical $\mathrm{CO}_{2}$. Environ. Sci. Technol. 2014, 48 (15), 86128619.

(23) Boek, E. S. ; Coveney, P. V.; Skipper, N. T. Molecular Modeling of Clay Hydration: A Study of Hysteresis Loops in the Swelling Curves of Sodium Montmorillonites. Langmuir 1995, 11 (12), 46294631.

(24) Boek, E. S. ; Coveney, P. V.; Skipper, N. T. Monte Carlo Molecular Modeling Studies of Hydrated Li-, Na-, and K-Smectites: Understanding the Role of Potassium as a Clay Swelling Inhibitor. J. Am. Chem. Soc. 1995, 117 (50), 1260812617. 
(25) Boek, E. S. Molecular Dynamics Simulations of Interlayer Structure and Mobility in Hydrated Li-, Na- and K-Montmorillonite Clays. Mol. Phys. 2014, 112, 14721483.

(26) Busch, A.; Alles, S.; Gensterblum, Y.; Prinz, D.; Dewhurst, D. N.; Raven, M. D.; Stanjek, H.; Krooss, B. M. Carbon dioxide storage potential of shales. Int. J. Greenhouse Gas Control 2008, 2, 297-308.

(27) Giesting, P.; Guggenheim, S.; Koster van Groos, A. F.; Busch, A. Interaction of carbon dioxide with Na-exchanged montmorillonite at pressures to 640 bar: Implications for $\mathrm{CO}_{2}$ sequestration. Int. J. Greenhouse Gas Control 2012, 8, 73-81.

(28) Romanov, V. N. Evidence of irreversible $\mathrm{CO}_{2}$ intercalation in montmorillonite. Int. J. Greenhouse Gas Control 2013, 81, 220-226.

(29) Schaef, H. T.; Loring, J. S.; Glezakou, V. A.; Miller, Q. R.; Chen, J.; Owen, A. T.; Lee, M. S.; Ilton, E. S.; Felmy, A. R.; McGrail, B. P.; Thompson, C. J. Competitive sorption of $\mathrm{CO}_{2}$ and $\mathrm{H}_{2} \mathrm{O}$ in 2:1 layer phyllosilicates. Geochim. Cosmochim. Acta 2015, 161, 248-257.

(30) Javadpour, F.; Fisher, D.; Unsworth, M. Nanoscale Gas Flow in Shale Gas Sediments. J. Can. Petrol. Technol. 2007, 46 (10), 55-61.

(31) Ross, D.J.K.; Bustin, R. M. The importance of shale composition and pore structure upon gas storage potential of shale gas reservoirs. Mar. Pet. Geol. 2009, 26 (6), 916-927.

(32) Liu, D.; Yuan, P.; Liu, H.; Li, T.; Tan, D.; Yuan, W.; He, H. High-pressure adsorption of methane on montmorillonite, kaolinite and illite. Appl. Clay Sci. 2013, 85, 25-30.

(33) Pusch, R. Clays and Nuclear Waste Management. In Handbook of Clay Science; 
2nd ed.; Bergaya, F.; Theng, B. K. G.; Lagaly, G., Eds.; Elsevier: Amsterdam, 2006; Vol. 1, pp 703716.

(34) Sellin, P.; Leupin, O. X. The Use of Clay as an Engineered Barrier in RadioactiveWaste Management-A Review. Clays Clay Miner. 2013, 61 (6), 477-498.

(35) Churakov, S. V. Mobility of Na and Cs on Montmorillonite Surface under Partially Saturated Conditions. Environ. Sci. Technol. 2013, 47, 98169823.

(36) Kumar, N.A.; Seidel, C. Polyelectrolyte Brushes with Added Salt. Macromolecules 2005, 38 (22), 9341-9350.

(37) Nair, A.K.N.; Uyaver, S.; Sun, S. Conformational Transitions of a Weak Polyampholyte. J. Chem. Phys. 2014, 141 (13), 134905.

(38) Kadoura, A.; Nair, A. K. N.; Sun, S. Adsorption of Carbon Dioxide, Methane and their Mixture by Montmorillonite in the Presence of Water. Microporous Mesoporous Mater. 2016, 225, 331-341.

(39) Kadoura, A.; Nair, A. K. N.; Sun, S. Molecular Dynamics Simulations of Carbon Dioxide, Methane, and their Mixture in Montmorillonite Clay Hydrates. J. Phys. Chem. C 2016, 120, 1251712529.

(40) Kadoura, A.; Nair, A. K. N.; Sun, S. Molecular Simulation Study of Montmorillonite in Contact with Variably Wet Supercritical Carbon Dioxide. J. Phys. Chem. C 2017, 121 (11), 6199-6208.

(41) Yang, Y.; Nair, A. K. N.; Sun, S. Molecular Dynamics Simulation Study of Carbon Dioxide, Methane, and Their Mixture in the Presence of Brine. J. Phys. Chem. B 2017, 121 (41), 9688-9698.

(42) Martin, M. G. MCCCS Towhee: A Tool for Monte Carlo Molecular Simulation. Mol. Simul. 2013, 39, 12121222. 
(43) Plimpton, S. Fast parallel algorithms for short-range molecular dynamics. J. Comput. Phys. 1995, 117, 1-19.

(44) Frenkel, D.; Smit, B. Understanding Molecular Simulation: From Algorithms to Applications; Academic Press: London, 2002.

(45) Cygan, R. T.; Liang, J. J.; Kalinichev, A. G. Molecular Models of Hydroxide, Oxyhydroxide, and Clay Phases and the Development of a General Force Field. J. Phys. Chem. B 2004, 108 (4), 1255-1266.

(46) Berendsen, H. J. C.; Grigera, J. R.; Straatsma, T. P. The Missing Term in Effective Pair Potentials. J. Phys. Chem. 1987, 91, 6269-6271.

(47) Åqvist, J. Ion-Water Interaction Potentials Derived from Free Energy Perturbation Simulations. J. Phys. Chem. 1990, 94 (21), 8021-8024.

(48) Koneshan, S.; Rasaiah, J. C.; LyndenBell, R. M.; Lee, S. H. Solvent Structure, Dynamics, and Ion Mobility in Aqueous Solutions at $25^{\circ} \mathrm{C}$. J. Phys. Chem. B 1998, 102 (21), 4193-4204.

(49) Errington, J. R.; Panagiotopoulos, A. Z. A Fixed Point Charge Model for Water Optimized to the Vapor-Liquid Coexistence Properties. J. Phys. Chem. B 1998, $102(38), 7470-7475$.

(50) J.-C. Liu; P. A. Monson Monte Carlo Simulation Study of Water Adsorption in Activated Carbon. Ind. Eng. Chem. Res. 2016, 45 (16), 5649-5656.

(51) Israelachvili, J. N. Adhesion forces between surfaces in liquids and condensable vapours. Surf. Sci. Rep. 1992, 14, 109-159.

(52) Myshakin, E. M.; Makaremi, M.; Romanov, V. N.; Jordan, K. D.; Guthrie, G. D. Molecular Dynamics Simulations of Turbostratic Dry and Hydrated Montmorillonite with Intercalated Carbon Dioxide. J. Phys. Chem. A 2014, 118 (35), 7454-7468. 
(53) Sposito, G.; Skipper, N. T.; Sutton, R.; Park, S.-H.; Soper, A. K.; Greathouse, J. A. Surface Geochemistry of the Clay Minerals. Proc. Natl. Acad. Sci. U.S.A. 1999, 96, 3358-3364.

(54) Brunauer, S.; Emmett, P. H.; Teller, E. Adsorption of Gases in Multimolecular Layers. J. Am. Chem. Soc. 1938, 60, 309-319.

(55) Marry, V.; Rotenberg, B.; Turq, P. Structure and Dynamics of Water at a Clay Surface from Molecular Dynamics Simulation. Phys. Chem. Chem. Phys. 2008, 10, 48024813.

(56) Holmboe, M.; Bourg, I.C. Molecular Dynamics Simulations of Water and Sodium Diffusion in Smectite Interlayer Nanopores as a Function of Pore Size and Temperature. J. Phys. Chem. C 2014, 118 (2), 1001-1013.

(57) Vanysek, P. Ionic Conductivity and Diffusion at Infinite Dilution. In CRC Handbook of Chemistry and Physics, 84th ed.; Lide, D. R., Ed.; CRC Press: Boca Raton, FL, 2003.

(58) Holz, M.; Heil, S. R.; Sacco, A. Temperature-Dependent Self-Diffusion Coefficients of Water and Six Selected Molecular Liquids for Calibration in Accurate ${ }^{1}$ H NMR PFG Measurements. Phys. Chem. Chem. Phys. 2000, 2, 47404742.

(59) Franks, F. Aqueous Solutions of Simple Electrolytes; Springer: New York, 1973; Vol. 3, p 472.

(60) Liu, P.; Harder, E.; Berne, B. J. On the Calculation of Diffusion Coefficients in Confined Fluids and Interfaces with an Application to the Liquid-Vapor Interface of Water. J. Phys. Chem. B 2004, 108, 65956602.

(61) Honorio, T.; Brochard, L.; Vandamme, M. Hydration phase diagram of clay particles from molecular simulations. Langmuir 2017, 33 (44), 12766-12776. 
Table 1: Approximate relative humidity $(\mathrm{RH})$ ranges and the corresponding hydration states/basal $d$-spacings observed experimentally and employed in our simulations for clay minerals in contact with water at $T=298.15 \mathrm{~K}$. The maximum deviation of the basal spacings obtained by swelling free-energy analysis/experiments from those used here was estimated at approximately $\pm 0.4 \AA$.

\begin{tabular}{lccc}
\hline \multirow{2}{*}{ Clay type } & RH $(\%)$ & \multicolumn{2}{c}{ hydration state/basal $d$-spacing } \\
\cline { 3 - 4 } & & Experiment $^{8}$ & Simulation $(\AA)$ \\
\hline Li-montmorillonite & $0-60$ & $1 \mathrm{~W}$ & 12.0 \\
& $60-100$ & $2 \mathrm{~W}$ & 15.0 \\
\hline Na-montmorillonite & $0-20$ & $0 \mathrm{~W}$ & 10.0 \\
& $20-60$ & $1 \mathrm{~W}$ & 12.0 \\
& $60-100$ & $2 \mathrm{~W}$ & 15.0 \\
\hline K-montmorillonite & $0-20$ & $0 \mathrm{~W}$ & 10.0 \\
& $20-100$ & $1 \mathrm{~W}$ & 12.0 \\
\hline Mg-montmorillonite & $0-20$ & $1 \mathrm{~W}$ & 12.0 \\
& $20-100$ & $2 \mathrm{~W}$ & 15.0 \\
\hline Ca-montmorillonite & $0-20$ & $1 \mathrm{~W}$ & 12.0 \\
& $20-100$ & $2 \mathrm{~W}$ & 15.0 \\
\hline Sr-montmorillonite & $0-40$ & $1 \mathrm{~W}$ & 12.0 \\
& $40-100$ & $2 \mathrm{~W}$ & 15.0 \\
\hline
\end{tabular}



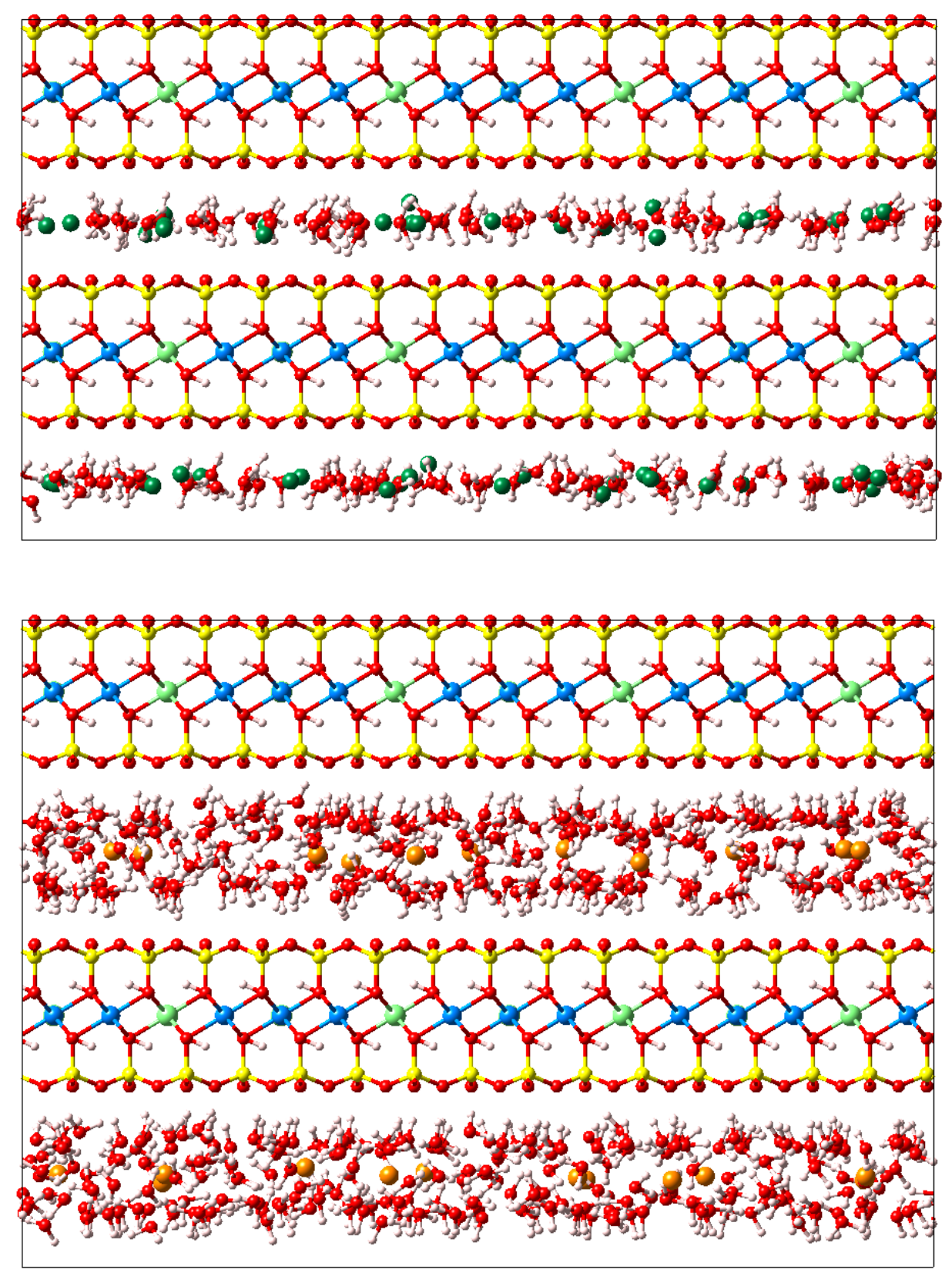

Figure 1: Equilibrium snapshots of K- (top) and Sr-montmorillonite (bottom) in contact with water $(\mathrm{RH}$ of $60 \%)$ at $T=298.15 \mathrm{~K}$. Color code: O, red; H, white; Si, yellow; Al, light blue; Mg, light green; C, black; K, dark green; Sr, orange. 

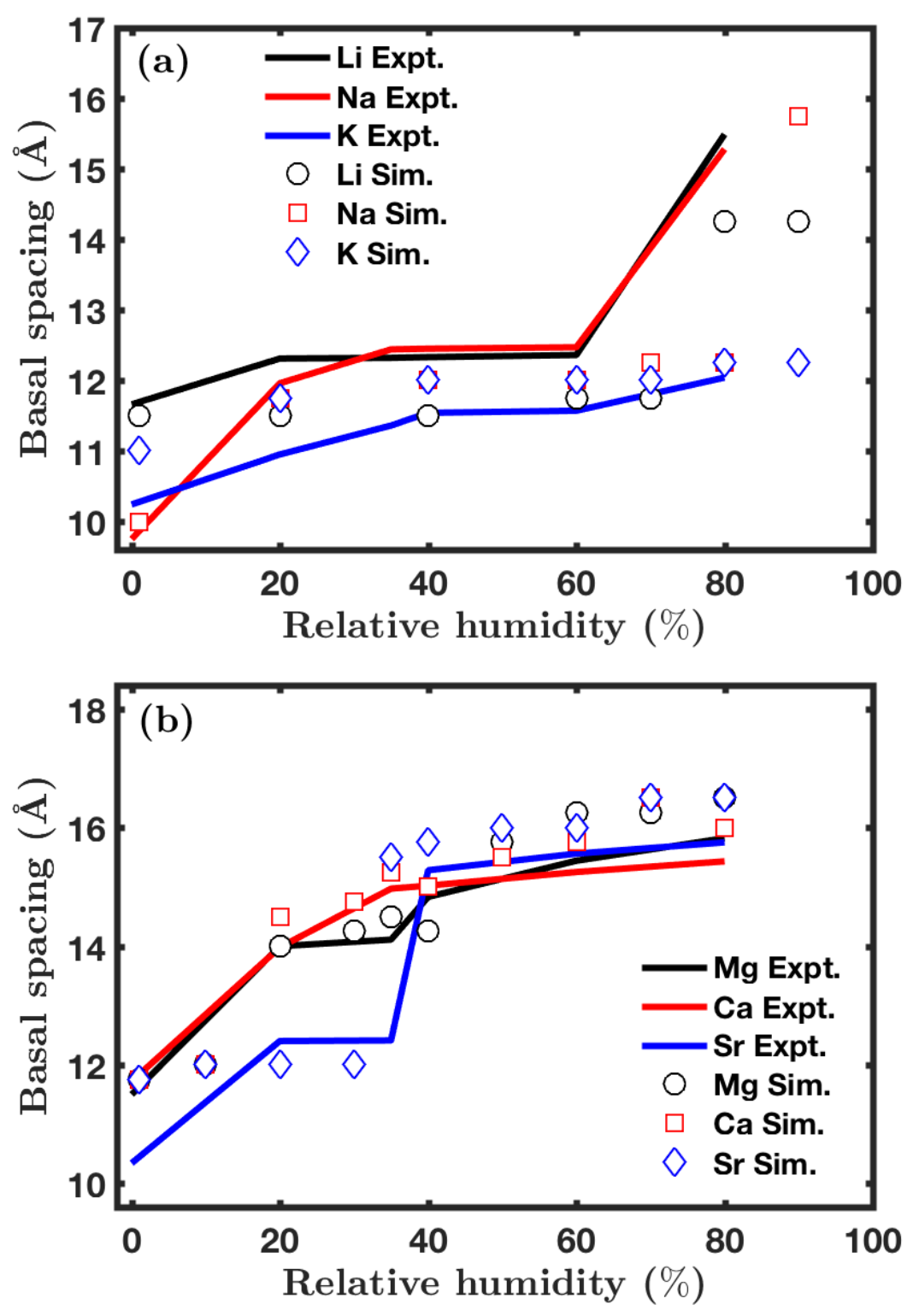

Figure 2: RH dependence of the basal $d$-spacing as computed from our GCMC simulations (symbols) and the corresponding experimental data ${ }^{8}$ (lines) for montmorillonite samples saturated with (a) monovalent and (b) divalent cations at $T=298.15 \mathrm{~K}$. For simulation, the maximum error was estimated at approximately $\pm 0.4 \AA$. 

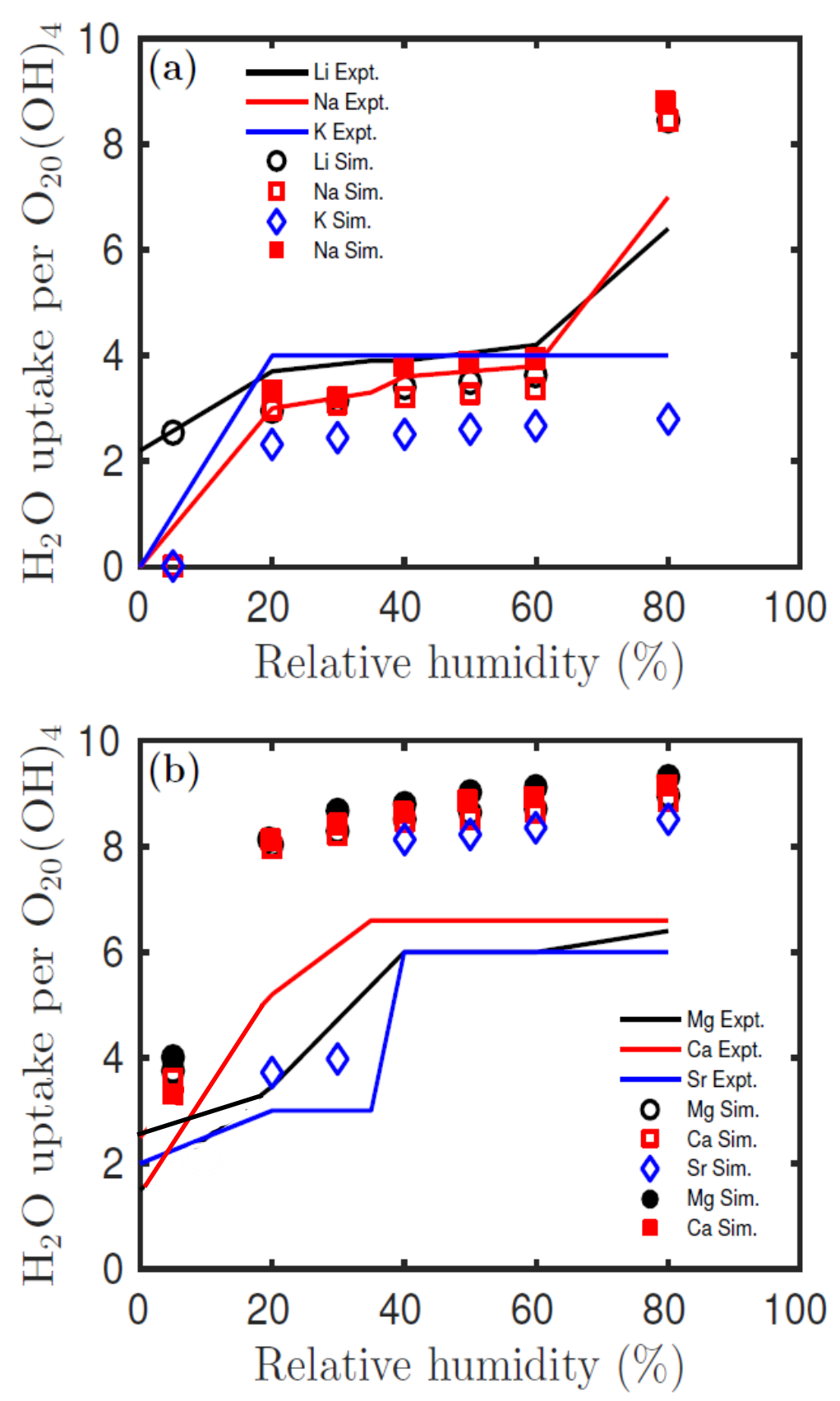

Figure 3: RH dependence of water uptake as computed from our GCMC simulations (open symbols) and the corresponding experimental data ${ }^{8}$ for montmorillonite samples saturated with (a) monovalent and (b) divalent cations at $T=298.15 \mathrm{~K}$. Error bars are smaller than the symbol size. The basal $d$-spacings are as given in Table 1. Simulation data of Teich-McGoldrick et al. ${ }^{21}$ are shown as solid symbols. 

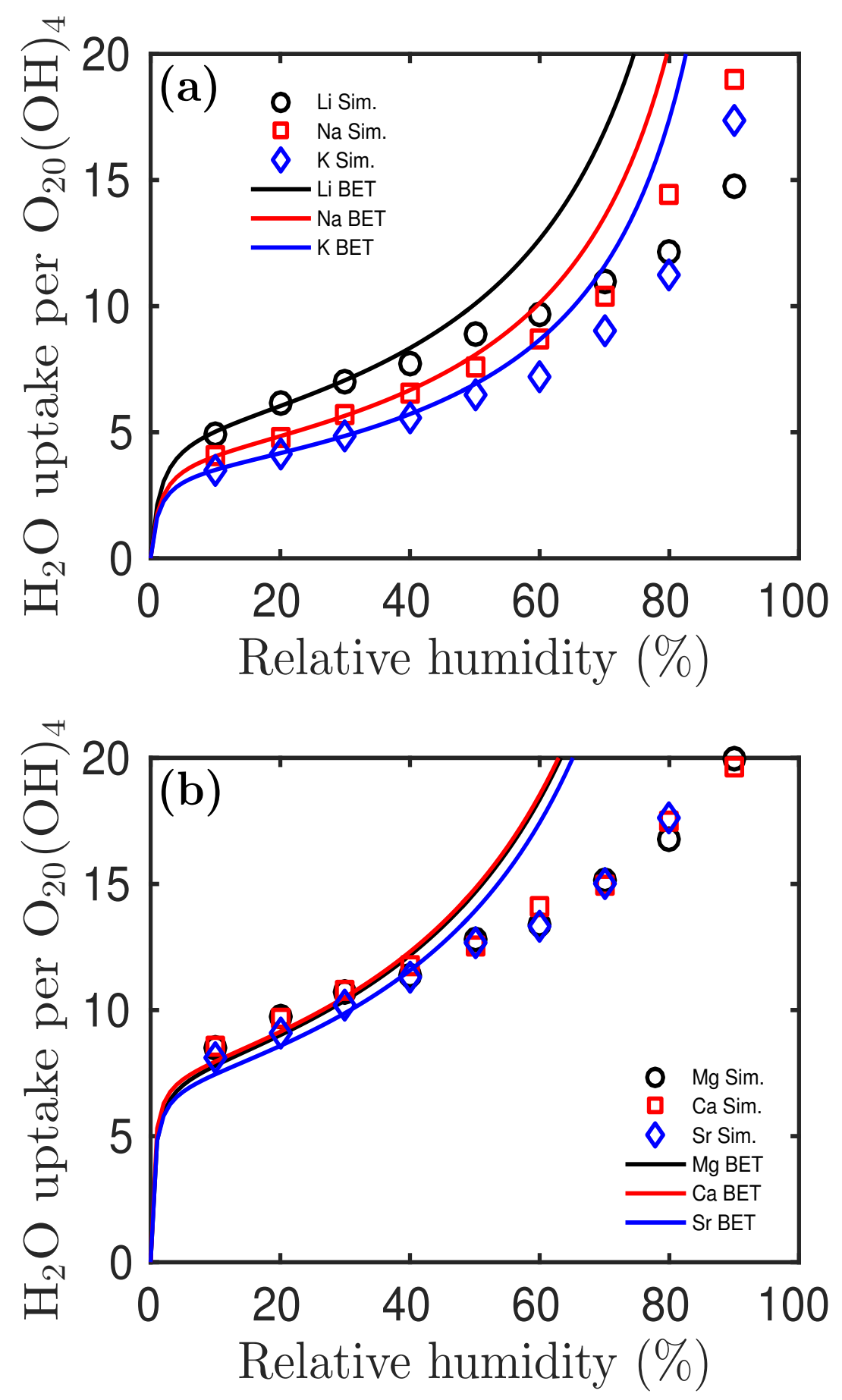

Figure 4: RH dependence of water uptake as computed from our GCMC simulations (symbols) and the corresponding BET model (lines) for montmorillonite samples saturated with (a) monovalent and (b) divalent cations at $T=298.15 \mathrm{~K}$. Error bars are smaller than the symbol size. The basal $d$-spacing is fixed at $65 \AA$. 

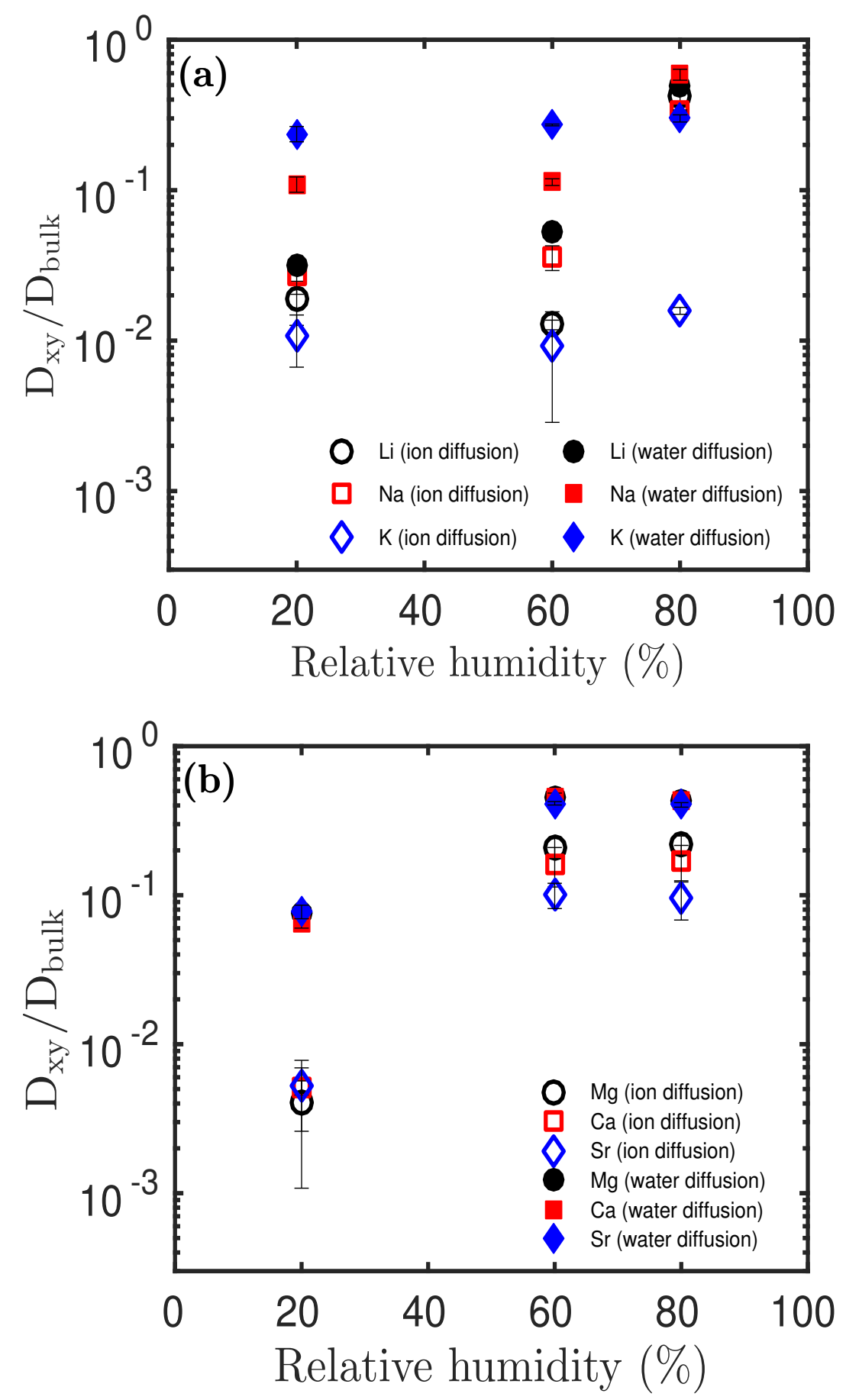

Figure 5: Normalized diffusion coefficients of interlayer ions (open symbols) and corresponding $\mathrm{H}_{2} \mathrm{O}$ (solid symbols) as a function of $\mathrm{RH}$ for montmorillonite samples saturated with (a) monovalent and (b) divalent cations at $T=298.15 \mathrm{~K}$. The basal $d$-spacings are as given in Table 1. 

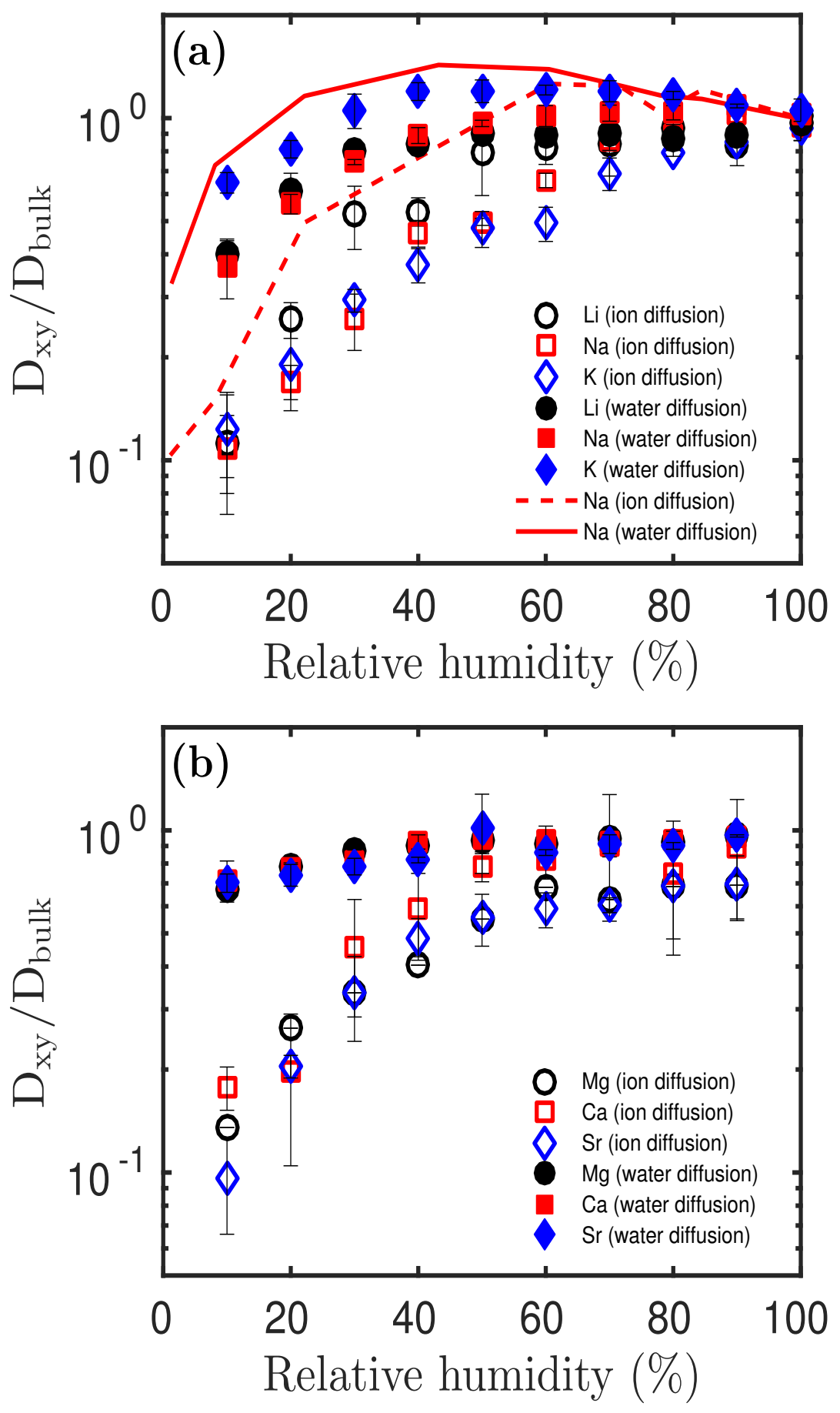

Figure 6: Normalized diffusion coefficients of ions (open symbols) and corresponding $\mathrm{H}_{2} \mathrm{O}$ (solid symbols) as a function of $\mathrm{RH}$ for montmorillonite samples saturated with (a) monovalent and (b) divalent cations at $T=298.15 \mathrm{~K}$. The basal $d$-spacing is fixed at $65 \AA$. Simulation data of Churakov ${ }^{35}$ obtained using SPC/E water model are shown as lines. 

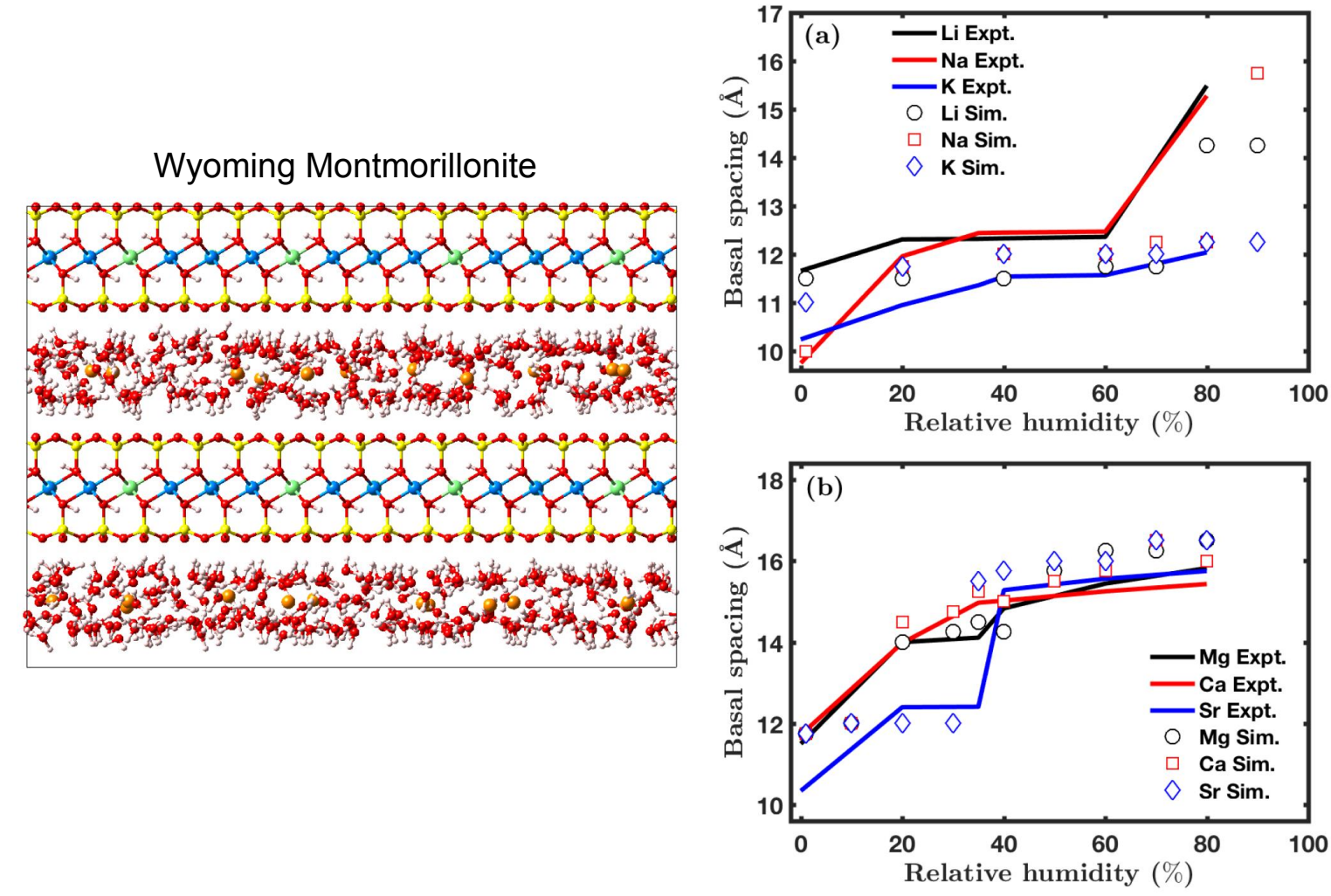

Table of Contents (TOC) Image 